\title{
ANH/MSD/CP-101078
}

10509

CONFERENCE PAPER

\section{EXPERIMENTAL METHODS AND DATA ANALYSIS FOR FLUCTUATION MICROSCOPY}

P. M. Voyles, Department of Physics, University of Illinois at Urbana-Champaign, 1110 West Green Street, Urbana, IL 61801

M. M. J. Treacy, NEC Research Institute, 4 Independence Way, Princeton, NJ 08540

J. Murray Gibson, Materials Science Division, Argonne National Laboratory, 9700 South Cass Avenue, Argonne, IL 60439-4838

H-C. Jin, Department of Materials Science and Engineering and Coordinated Science Laboratory, University of Illinois, Urbana, IL 61801

J. R. Abelson, Department of Materials Science and Engineering and Coordinated Science Laboratory, University of Illinois, Urbana, IL 61801

January, 2000

The submitted manuscript has been created
by the University of Chicago as Operator of
Argonne National Laboratory ("Argonne")
under Contract No. W-31-109-ENG-38 with
the U.S. Department of Energy. The U.S.
Government retains for itself, and others
acting on its behalf, a paid-up, non exclusive,
irrevocable worldwide license in said article to
reproduce, prepare derivative works, distribute
copies to the public, and perform publicly and
display publicly, by or on behalf of the
Government.

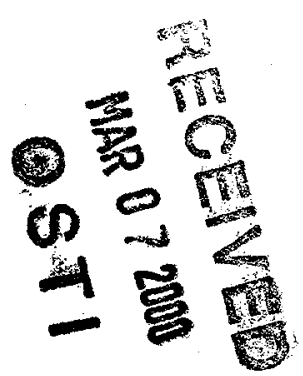

Distribution:

1-2. PRS

3. J. M. Gibson

4. L. E. Rehn

5. J. Coble

6. File

Conference Paper to be published in the proceedings of the Materials Research Society's Fall 1999 Meeting.

*Work supported by the U.S. Department of Energy through the Basic Energy SciencesMaterials Sciences under contract No. W-31-109-Eng-38. 


\section{DISCLAIMER}

This report was prepared as an account of work sponsored by an agency of the United States Government. Neither the United States Government nor any agency thereof, nor any of their employees, make any warranty, express or implied, or assumes any legal liability or responsibility for the accuracy, completeness, or usefulness of any information, apparatus, product, or process disclosed, or represents that its use would not infringe privately owned rights. Reference herein to any specific commercial product, process, or service by trade name, trademark, manufacturer, or otherwise does not necessarily constitute or imply its endorsement, recommendation, or favoring by the United States Government or any agency thereof. The views and opinions of authors expressed herein do not necessarily state or reflect those of the United States Government or any agency thereof. 


\section{DISCLAIMER}

Portions of this document may be illegible in electronic image products. Images are produced from the best available original document. 
CONFERENCE PAPER

\section{EXPERIMENTAL METHODS AND DATA ANALYSIS FOR FLUCTUATION MICROSCOPY}

P. M. Voyles, Department of Physics, University of Illinois at Urbana-Champaign, 1110 West Green Street, Urbana, IL 61801

M. M. J. Treacy, NEC Research Institute, 4 Independence Way, Princeton, NJ 08540

J. Murray Gibson, Materials Science Division, Argonne National Laboratory, 9700 South Cass Avenue, Argonne, IL 60439-4838

H-C. Jin, Department of Materials Science and Engineering and Coordinated Science Laboratory, University of Illinois, Urbana, IL 61801

J. R. Abelson, Department of Materials Science and Engineering and Coordinated Science Laboratory, University of Illinois, Urbana, IL 61801

January, 2000

The submitted manuscript has been created
by the University of Chicago as Operator of
Argonne National Laboratory ("Argonne")
under Contract No. W-31-109-ENG-38 with
the U.S. Department of Energy. The U.S.
Government retains for itself, and others
acting on its behalf, a paid-up, non exclusive,
irrevocable worldwide license in said article to
reproduce, prepare derivative works, distribute
copies to the public, and perform publicly and
display publicly, by or on behalf of the
Government.

Conference Paper to be published in the proceedings of the Materials Research Society's Fall 1999 Meeting

${ }^{*}$ Work supported by the U.S. Department of Energy through the Basic Energy SciencesMaterials Sciences under contract No. W-31-109-Eng-38. 


\title{
Experimental Methods and Data Analysis for Fluctuation Microscopy
}

\author{
P. M. Voyles, ${ }^{* \dagger}$ M. M. J. Treacy, ${ }^{\dagger}$ J. M. Gibson, ${ }^{\ddagger}$ H-C. Jin, ${ }^{* *}$ and J. R. Abelson ${ }^{* *}$ \\ *Dept. of Physics, University of Illinois, Urbana, IL 61801, pvoyles@uiuc.edu \\ ${ }^{\dagger}$ NEC Research Institute, Princeton, NJ 08540 \\ ${ }^{\ddagger}$ Argonne National Laboratory, Argonne, IL 60439 \\ ${ }^{* *}$ Dept. of Materials Science and Engineering and Coordinated Science Laboratory, Univer- \\ sity of Illinois, Urbana, IL 61801
}

\begin{abstract}
We have developed a new electron microscopy technique called fluctuation microscopy which is sensitive to medium-range order in disordered materials. The technique relies on quantitative statistical analysis of low-resolution dark-field electron micrographs. Extracting useful information from such micrographs involves correcting for the effects of the imaging system, incoherent image contrast caused by large scale structure in the sample, and the effects of the foil thickness.
\end{abstract}

\section{INTRODUCTION TO FLUCTUATION MICROSCOPY}

It has long been recognized that the pair distribution function $g_{2}(r)$ yielded by kinematic diffraction is an insufficient characterization of the structure of disordered materials such as amorphous semiconductors. Several computer simulations of disordered materials have been produced which have identical $g_{2}(r)$, but different structure at medium range $[1,2]$. Moving beyond the pair correlation function has proved difficult, however. Experimental techniques such as Raman scattering, near-edge x-ray absorption fine structure, nuclear magnetic resonance, and electrical noise measurements have offered tantalizing glimpses of structural differences at medium range, but remain difficult to interpret in terms of purely structural models since such signals depend in a complicated way on the details of the interatomic potential. Fluctuation microscopy uses high-energy electrons as a probe, so it does not suffer from those limitations.

Fluctuation microscopy depends on the statistical analysis of hollow-cone dark-field (HCDF) micrographs taken at deliberately low resolution, $\sim 1 \mathrm{~nm}$. In this limit, we can ignore the effects of microscope aberrations and view the image as a map of the diffracted intensity from mesoscopic volumes of the sample, the size of which is set by the resolution, which is in turn controlled by the size of the objective aperture. There are then two parameters which characterize the imaging conditions: the magnitude of the dark-field scattering vector $k$ and the objective aperture diameter $Q$. We compute the variance of the "diffraction map" image as a function of $k$ and $Q$

$$
V(k, Q)=\frac{\left\langle I^{2}(k, Q, \mathbf{r})\right\rangle}{\langle I(k, Q, \mathbf{r})\rangle^{2}}-1
$$

where \langle\rangle denotes averaging over the image position coordinate $\mathbf{r}$.

To understand in a qualitative way why the variance is sensitive to medium-range order (MRO), consider two samples: one a homogeneous random assortment of atoms with no MRO and the other a heterogeneous material with small, randomly-oriented ordered clusters. 
Set $Q$ so the mesoscopic volume is approximately the same size as those clusters. All of the mesoscopic volumes of the random sample will be statistically the same, so the diffracted intensity from each volume will be the same, and the image will have a low variance. In the heterogeneous sample, some of the clusters will be oriented near a Bragg condition and diffract strongly and others will not. These differences in diffracted intensity lead to an image with large variance. In general, a large image variance indicates some form of MRO.

By varying the imaging conditions $k$ and $Q$ we can obtain information about the character of any MRO present. Varying $k$ at constant $Q$ is called variable coherence microscopy and gives information about the structure and degree of ordering inside any ordered regions. Varying $Q$ at constant $k$ is called variable resolution microscopy and gives information about the size of the ordered regions. Only variable coherence microscopy has been experimentally implemented so far.

The quantitative information fluctuation microscopy provides is complicated and still incompletely understood, but we know that it depends on the four-body pair-pair correlation function $g_{4}\left(r_{1}, r_{2}, r, \theta\right)$, where $r_{1}$ and $r_{2}$ define one pair lengths, $r$ is the distance between pairs, and $\theta$ is their relative angle [3]. It has been shown that the pair-pair correlation function contains more information about MRO than $g_{2}(r)[4]$.

Fluctuation microscopy has been used to show that thin films of amorphous semiconductors contain more MRO than can be explained by the continuous random network, and that that MRO is reduced on thermal annealing [1] (and for hydrogenated amorphous silicon by exposure to light [5]). This has lead to the development of the paracrystalline theory of the structure of as-deposited amorphous thin films [6]. In this paper we focus on recent advances in the variable coherence experimental method and data analysis.

\section{RECOVERING THE TRUE ELECTRON STATISTICS}

The advent of charge-coupled device (CCD) cameras and other linear devices for recording electron images has made statistical techniques such as fluctuation microscopy possible. However, care must still be taken when analyzing such images. For fluctuation microscopy, we must carefully correct for the modulation transfer function (MTF) of our CCD camera.

The MTF is the reciprocal-space function which connects the measured electron intensity with the intensity incident on the device:

$$
I_{\text {measured }}(\kappa)=M T F(\kappa) I_{\text {incident }}(\kappa)
$$

where $\kappa$ is the Fourier transform coordinate of the image, not a scattering vector. Since Parseval's theorem connects the variance and the power spectrum $P(\kappa)$ of the image, it is simple for us to correct for the MTF by

$$
V=\int 2 \pi \kappa^{2} \frac{P(\kappa)}{|M T F(\kappa)|^{2}} d \kappa
$$

We have measured the MTF of our Gatan MSC Model 794 CCD at an accelerating voltage of $200 \mathrm{kV}$ using the stochastic image method as given by Zuo [7]. Daberkow et. al. have noted that this technique is sensitive to aliasing in the calculation of the stochastic image power spectrum [8]. We have investigated this by the simple expedient of resampling the acquired images, expanding each original pixel into 4 pixels, then 8 , then 16 so that the discrete Fourier transform approaches the ideal continuous transform. 


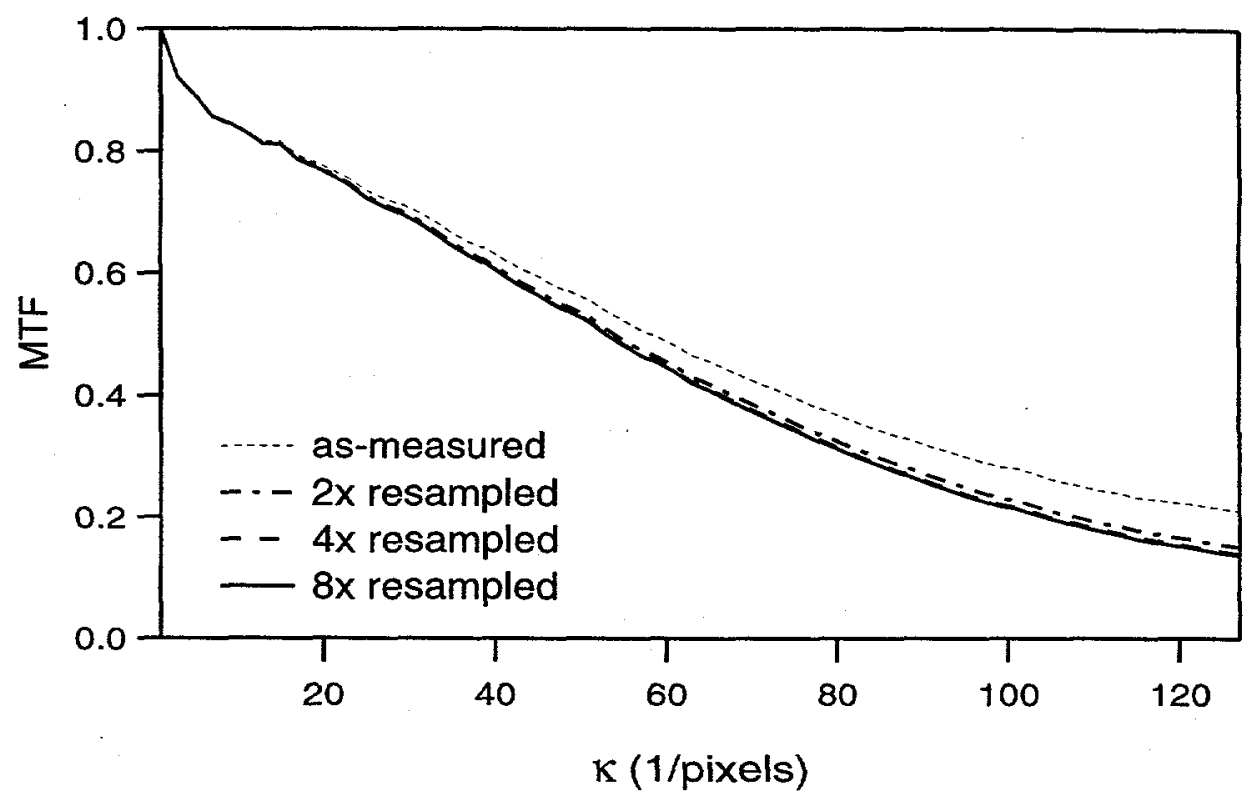

Figure 1: The MTF of the Gatan MSC 794 camera as measured by analyzing stochastic images as measured, then with varying resampling before computation of the Fourier transforms.

Figure 1 shows the results of this analysis. The effects of the resampling are most dramatic at high frequency. At the Nyquist frequency of 1281 /pixels, the MTF without resampling is 0.209 , which is similar to previous reports for other cameras $[7,8,9]$. However, the transfer is reduced to 0.15 at $2 \times$ resampling, and 0.139 and 0.136 at $4 \times$ and $8 \times$ respectively. The small reduction between $4 \times$ and $8 \times$ makes it unlikely that the MTF would be further suppressed by additional resampling. We conclude that $4 \times$ resampling is sufficient and necessary for accurate measurement of the MTF by the stochastic image method.

\section{REMOVING INCOHERENT VARIANCE}

So far we have assumed that all of the sources of variance are due to the microstructure of the material. This is clearly not the case, as variance (image contrast) can be caused by a variety of other mechanisms in real samples. If there are macroscopic features in the sample, such as thickness variations, bends, ridges, or holes, these will contribute massthickness variance. Non-uniform illumination will also contribute variance. What these have in common is that the contrast mechanism is incoherent. Incoherent variance contributes a $k$-independent systematic error to $V(k)$ which is different for every area of the sample.

We remove incoherent variance from the analysis in two ways. First, we Fourier filter the images [6]. This is easily implemented by adjusting the limits of integration in Equation 3. This is particularly good at removing the effects of non-uniform illumination, which are low in spatial frequency. Macroscopic features in the image are more difficult to remove in this way, as they can contribute power over a range of frequencies.

We correct for variance due to inhomogeneities by direct measurement. At large enough scattering vector $\left(k=14.0 \mathrm{~nm}^{-1}\right.$ for $\mathrm{Si}$ ), HCDF images become incoherent [10]. (This is the 
optical conjugate of $Z$-contrast microscopy using a high-angle annular dark field detector in a STEM.) In this limit, the type of variance described in the first section due to differences in coherent diffraction is suppressed, leaving only variance due to mass-thickness. We acquire several such high-angle images for each area of the film investigated, and subtract the average of their variances from the measured variance at lower $k$. This effectively removes the $k$ independent systematic error, although it only works if there is negligible specimen drift during the acquisition of the entire series of images. The ability to measure the incoherent variance is the primary advantage of working in HCDF instead of tilted dark field.

\section{THICKNESS EFFECTS}

Foil thickness also plays a role in the experimental variance. In order to compare the variance of different samples, rather than the same sample before and after some treatment as we have done previously, we need to understand and correct for the effects of thickness.

We have measured the properties of a set of six films of varying thickness in order to investigate these effects. The samples are a-Si: $\mathrm{H}$ thin films deposited by reactive magnetron sputtering of a Si target in an Ar plasma at a substrate temperature of $230^{\circ} \mathrm{C}$ at a rate of $\sim 0.29 \mathrm{~nm} / \mathrm{sec}$. With $\mathrm{H}_{2}$ in the discharge, this experimental system routinely produces a-Si:H films whose properties are identical to those grown by optimized plasma-CVD [11]. The films are deposited on top of the commercial Al foil and Corning 7059 glass simultaneously. The thickness of the films was determined by fitting the optical transmittance and reflectance of the films on glass measured with a Cary 5000 dual-beam spectrophotometer using the Tauc-Lorenz model established by Jellison and Modin [12]. TEM samples were prepared by etching off the Al substrate in $20 \%$ hydrochloric acid solution then catching the free-standing film on a support grid. Variable coherence measurements were performed in a Hitachi H900O TEM with a computer-controlled hollow cone unit and automated image acquisition system operated at $200 \mathrm{kV}$ with an objective aperture which yields an image resolution of $\sim 1.5$ $\mathrm{nm}$. Each $V(k)$ trace is the average of 10-12 areas on the film, requiring $\sim 250$ images. Error bars are one standard deviation of the mean.

First, we need a convenient method to measure film thickness in the TEM. If we assume a Poisson distribution for the probability of $n$ scattering events and the average BF intensity $\left\langle I_{B F}\right\rangle /\left\langle I_{0}\right\rangle$ is due to unscattered electrons, we predict that $\left\langle I_{B F}\right\rangle /\left\langle I_{0}\right\rangle$ decays exponentially with increasing film thickness $[13],\left\langle I_{B F}\right\rangle /\left\langle I_{0}\right\rangle=\exp \left(-t / \Lambda_{B F}\right) . I_{0}$ is the intensity of the beam, and \langle\rangle indicates averaging over the image. Figure 2(a) shows $\left\langle I_{B F}\right\rangle /\left\langle I_{0}\right\rangle$ for our samples, from which we determine that for a-Si:H $\Lambda_{B F}=106 \pm 3 \mathrm{~nm}$ under the imaging conditions described above. This allows us to measure in situ the thickness of such films so we can correct the experimental variance.

Next, we examine the thickness scaling of the denominator of Equation 1, the average DF intensity. If the average DF intensity $\left\langle I_{D F}\right\rangle /\left\langle I_{0}\right\rangle$ is due to electrons scattered once, $\left\langle I_{D F}\right\rangle /\left\langle I_{0}\right\rangle=C_{1}\left(t / \Lambda_{D F}(k)\right) \exp \left(-t / \Lambda_{D F}(k)\right)$, where $C_{1}$ is a scaling constant. Figure 2(b) shows $\left\langle I_{D F}\right\rangle /\left\langle I_{0}\right\rangle$ and fits to this functional form for our samples. $\Lambda_{D F}\left(3.3 \mathrm{~nm}^{-1}\right)=125 \pm 9$ $\mathrm{nm}, \Lambda_{D F}\left(4.5 \mathrm{~nm}^{-1}\right)=140 \pm 15 \mathrm{~nm}$, and $\Lambda_{D F}\left(5.75 \mathrm{~nm}^{-1}\right)=164 \pm 5 \mathrm{~nm}$.

The last piece of the thickness scaling of $V$ is the second intensity moment $\left\langle I^{2}\right\rangle$. If the sample consists of ordered clusters, the number of clusters increases linearly with thickness. If the clusters are uncorrelated, the variance of their scattered intensity increases linearly with the number of clusters, so $\left\langle I^{2}\right\rangle=C_{2}(k)\left(t-t_{0}\right) . t_{0}$ is $\sim 5 \mathrm{~nm}$, which is consistent with the presence of a thermal oxide layer on the Si surfaces. $C_{2}(k)$ will be determined by the size 

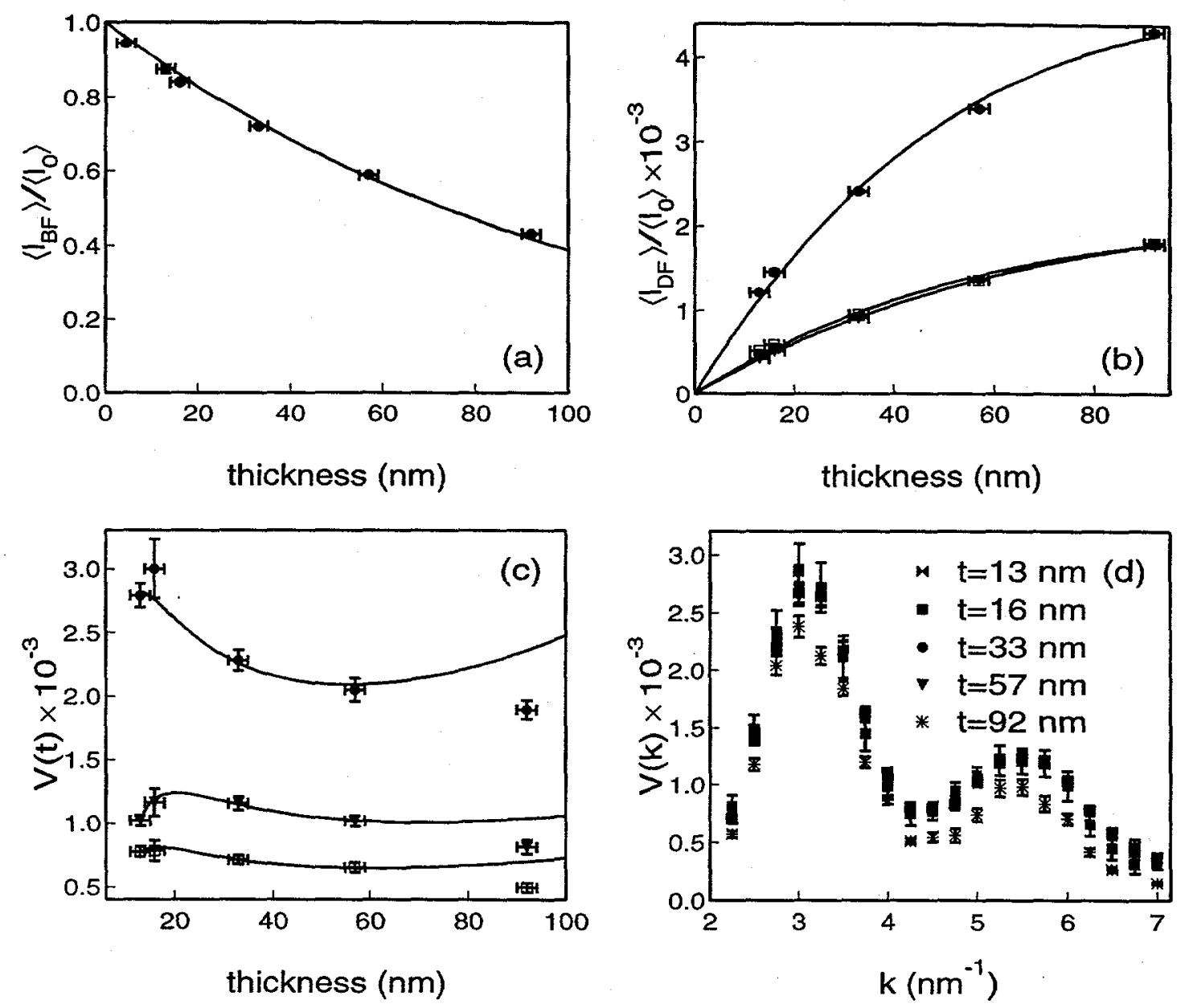

Figure 2: (a) Average BF transmission, (b) average DF transmission, (c) $V(t)$ for select $k$, and (d) $V(k)$ for films with various thickness, scaled to $t=20 \mathrm{~nm}$. In (b) and (c), $\bullet$ is $k=3.0 \mathrm{~nm}^{-1}, \square$ is $k=4.5 \mathrm{~nm}^{-1}$, and $\nabla$ is $k=5.75 \mathrm{~nm}^{-1}$.

of the ordered clusters, which we hope to measure directly by variable resolution microscopy, and the structure factor of the clusters.

Substituting into Equation 1, we predict that $V$ as a function of $t$ should be

$$
V(t)=\frac{C_{2}\left(t-t_{0}\right)}{\left(\frac{C_{1} t}{\Lambda_{D F}}\right)^{2} e^{-2 t / \Lambda_{D F}}}
$$

with two adjustable parameters $C_{2}$ and $t_{0}$, and $\Lambda_{D F}$ and $C_{1}$ measured by fitting $\left\langle I_{D F}\right\rangle /\left\langle I_{0}\right\rangle$. Figure 2(c) shows $V(t)$ data for a variety of $k$ and fits to Equation 4.

The fit clearly fails for $t>\Lambda_{D F} / 2$. Since $\left\langle I_{D F}\right\rangle$ is fit well over the full range considered, the failure must be due to a sublinear dependence of $\left\langle I^{2}\right\rangle$ on $t$ at large $t$. This may be due to small-angle diffuse scattering. Such scattering would tend to smooth the image intensity toward the mean, reducing $\left\langle I^{2}\right\rangle$, but would still be accepted by the objective aperture and not cause deviation in $\left\langle I_{D F}\right\rangle$. 
Figure 2(d) shows $V(k)$ for films of varying thickness all scaled to a thickness of $t=20 \mathrm{~nm}$ using Equation 4 and parameters extracted by fitting. With the exception of the thickest film data, all the curves are the same within experimental error, demonstrating that we can accurately correct for the effects of thickness on $V(k)$. It is necessary to repeat the measurement of $V(k)$ for various thicknesses to extract the relevant parameters if one wishes to apply this correction method to a $k$ outside the range measured here or to a material other than a-Si.

\section{CONCLUSIONS}

Fluctuation microscopy is a powerful new tool for the investigation of MRO in disordered materials. We have detailed the experimental methods and data analysis necessary to reliably compare samples prepared by different means to different thicknesses. This involves deconvolving the MTF of the imaging system, Fourier filtering the resulting images, correcting for the effects of incoherent variance by high-angle HCDF imaging, and correcting for foil thickness.

\section{ACKNOWLEDGEMENTS}

P. M. V. thanks the National Science Foundation for support under grant DMR 97-03906.

\section{REFERENCES}

1. J. M. Gibson and M. M. J. Treacy, Phys. Rev. Letts. 78, 1074 (1997).

2. N. Mousseau and L. J. Lewis, Phys. Rev. Letts. 78, 1484 (1997).

3. J. M. Gibson and M. M. J. Treacy, to be published in Ultramicroscopy.

4. P. M. Voyles, J. M. Gibson, and M. M. J. Treacy, to be published in J. Electron Microscopy.

5. J. M. Gibson, M. M. J. Treacy, P. M. Voyles, H-C. Jin, and J. R. Abelson, Appl. Phys. Letts. 73, 3093 (1998).

6. M. M. J. Treacy, J. M. Gibson, and P. J. Keblinski, J. Non-Cryst. Solids 231, 99 (1998).

7. J. M. Zuo, Ultramicroscopy 66, 21 (1996).

8. I. Daberkow, K.-H. Hermann, L. Liu, W. D. Rau, and H. Tietz, Ultramicroscopy 64, 35 (1996).

9. O. L. Krivanek and P. E. Mooney, Ultramicroscopy 49, 95 (1993).

10. M. M. J. Treacy and J. M. Gibson, Ultramicroscopy 52, 31 (1993).

11. M. Pinarbasi, N. Maley, A. M. Myers, and J. R. Abelson, Thin Solid Films 171, 217 (1989); M. Pinarbasi, M. J. Kushner, and J. R. Abelson, J. Appl. Phys. 68, 2255 (1990).

12. G. E. Jellison, Jr. and F. A. Modin, Appl. Phys. Lett. 69, 371 (1996).

13. L. A. Freeman, A. Howie, A. B. Mistry, and P. H. Gaskell in The Structure of Non-Cryst. Mats. edited by P. H. Gaskell (Taylor \& Francis, London, 1976) p. 245. 